\title{
Rough surface scattering at low-grazing incidence: A dedicated model
}

\author{
M. Saillard ${ }^{1}$ and G. Soriano ${ }^{2}$ \\ Received 31 December 2010; revised 13 July 2011; accepted 18 July 2011; published 19 October 2011. \\ [1] The rough surface radar cross section becomes very small at grazing incidence, \\ since most part of the energy travels in the forward direction. We build a rigorous model \\ that can estimate it accurately. It is based on a specific integral formalism, and applies \\ to both low-grazing incidence and scattering angles. Reference data are produced to test \\ the validity of approximate models, and a technique for extrapolation is detailed.
}

Citation: Saillard, M., and G. Soriano (2011), Rough surface scattering at low-grazing incidence: A dedicated model, Radio Sci., 46, RS0E13, doi:10.1029/2010RS004641.

\section{Introduction}

[2] The way to compute the non-grazing radar cross section (RCS) from a random rough surface separating two homogeneous media without any simplifying assumption appears in the work of Wagner et al. [1997] and is detailed by Tsang et al. [2001]. It is based on the numerical solution of a set of boundary integral equations [Poggio and Miller, 1973; Colton and Kress, 1983] for a deterministic rough surface of finite area. A plane wave cannot be the incident field, because of edge effects: a tapered polarized beam [Toporkov et al., 1999; Braunisch et al., 2000] with a Gaussian-like footprint on the surface is the reference method in order to bound to the finite area of the rough surface the numerical support of the integral equations unknowns, namely the tangential components of the total electromagnetic field. Statistical RCS is obtained through Monte Carlo average.

[3] The RCS from a rough surface becomes very small at low-grazing incidence (LGI), since most part of the energy travels in the forward direction. In the work of Spiga et al. [2008], we have presented the Grazing Method of Moments (GMoM), a different but rigorous approach dedicated to the time harmonic electromagnetic wave scattering from rough surfaces under grazing illumination. The rough surface is represented by a bounded perturbation of a plane and is enlightened by a plane wave. The integral equations are built on specific unknowns and right hand sides that permit edge effects to be avoided. In the work of Soriano et al. [2010], the implementation of the GMoM for two-dimensional problems is detailed, and numerical results on one-dimensional (1D) Gaussian surfaces in Optics and 1D ocean-like surfaces at microwave frequencies are shown. Note that several other methods have been considered to eliminate the tapered wave requirement in rough surface scattering. They are summarized in the introduction of Spiga et al. [2008]. Plane wave

\footnotetext{
${ }^{1}$ Laboratoire de Sondage Electromagnétique de l'Environnement Terrestre, Université du Sud Toulon-Var, La Garde, France.

${ }^{2}$ Sondage ElectroMagnétique et Optique, Institut Fresnel, Aix-Marseille Université, Marseille, France.

Copyright 2011 by the American Geophysical Union. 0048-6604/11/2010RS004641
}

illuminating a bounded perturbation of the plane appears in the work of West [2003] and Zhao et al. [2005], but in those papers, edge effects are avoided thanks to resistive loadings. Also, in the work of Zhao et al. [2005], an image theory involving the half plane Green's function is addressed. The GMoM differs from those two pioneering approaches in that sense that first, the physical boundary condition on the rough surface is left untouched, and second, it only makes use of classical integral operators, built on the free space Green's function.

[4] This paper is organized as follows. Below this introduction, the boundary integral formalism is presented in section 2: after classical theory is recalled, integral equations are modified for numerical solution with an incident plane wave. Also, lossy dielectrics and conductors dielectric are given a specific treatment through an impedance boundary condition. In spite of appearances, the scattered field theoretically shows at both LGI and low-grazing scattering angles (LGS) a very simple and general behavior. This behavior, and how to enforce it numerically, is underlined in section 3, while in section 4, a technique for extrapolation, from one low-grazing angle to another, is suggested. Then in section 5, the GMoM is applied to two-dimensional (2D) perfectly conducting surfaces in the $\mathrm{HH}$ polarization case (both the incident electric field and the scattered electric field of interest are normal to the incidence plane). New numerical results are presented for $2 \mathrm{D}$ surfaces with Gaussian and ocean spectra.

\section{Boundary Integral Formalism}

[5] The study is time harmonic at pulsation $\omega$, with a time dependence $e^{-i \omega t}$ assumed. We start by considering an infinite rough surface separating vacuum as upper medium from a semi-infinite dielectric homogeneous lower medium, illuminated by a wave impinging from vacuum. The surface $\Sigma$ is a perturbation of the $(x O y)$ plane in the right Cartesian coordinate $(x, y, z)$ system with the $z$ axis directed toward vacuum (Figure 1). The surface height is given by equation $z=h(\mathbf{r})=h(x, y)$, and $\hat{\mathbf{n}}$ denotes its unit normal vector directed upward. If $\theta_{0}$ denotes the incidence angle, counted as indicated on Figure 1, then the incident field is low grazing when 


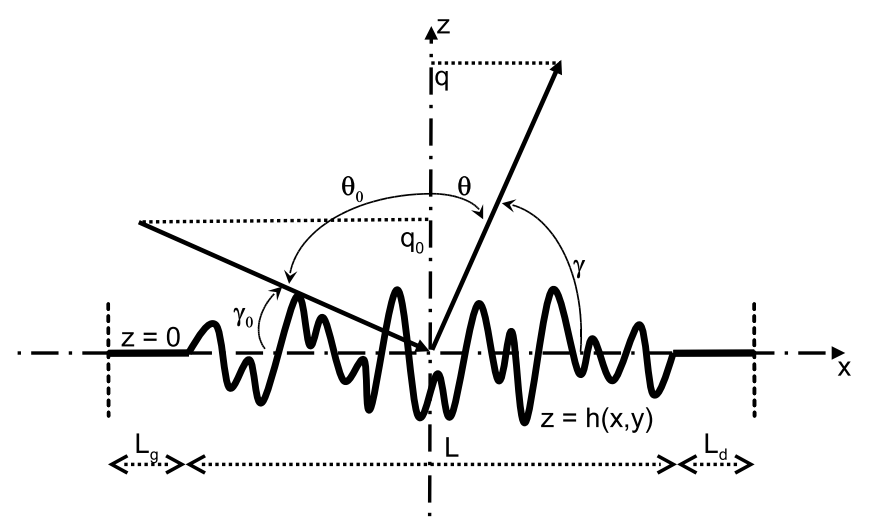

Figure 1. The bounded perturbation of the plane model for low-grazing incidence angles wave scattering from rough surfaces.

$\theta_{0} \rightarrow \pm \pi / 2$. The grazing angle can be defined as $\gamma_{0}=\frac{\pi}{2}-$ $\left|\theta_{0}\right| \rightarrow 0$ but we prefer to consider $q_{0}=K_{0} \cos \theta_{0}=K_{0} \sin \gamma_{0}$ that shares the same behavior. $K_{0}=\omega \sqrt{\varepsilon_{0} \mu_{0}}=\frac{2 \pi}{\lambda}$ is the wave number for the vacuum and $q_{0}$ is the positive $z$ component of the incident wave vector $\mathbf{k}_{0}-q_{0} \hat{\mathbf{z}}$. At the end, we are mainly concerned by the far field. Denoting $\mathbf{k}+q \hat{\mathbf{z}}$ the scattering wave vector in air at angle $\theta$ counted from normal or $\gamma=$ $\pi / 2-|\theta|$ from grazing, the LGS limit corresponds to $\gamma \rightarrow 0$ or $q=K_{0} \cos \theta=K_{0} \sin \gamma \rightarrow 0$.

[6] Following the classical Stratton-Chu theory [Poggio and Miller, 1973; Colton and Kress, 1983], the tangential components of the total fields on the rough surface $\hat{\mathbf{n}} \times \mathbf{E}$ and $\hat{\mathbf{n}} \times \mathbf{H}$ are the solution of integral equations for the air

$$
\begin{aligned}
& \left(\frac{1}{2}+M_{0}\right) \hat{\mathbf{n}} \times \mathbf{H}-\frac{i}{\omega \mu_{0}} P_{0} \hat{\mathbf{n}} \times \mathbf{E}=\hat{\mathbf{n}} \times \mathbf{E}^{i} \\
& \left(\frac{1}{2}+M_{0}\right) \hat{\mathbf{n}} \times \mathbf{E}+\frac{i}{\omega \varepsilon_{0}} P_{0} \hat{\mathbf{n}} \times \mathbf{H}=\hat{\mathbf{n}} \times \mathbf{H}^{i}
\end{aligned}
$$

and for the scattering medium

$$
\begin{aligned}
& \left(\frac{1}{2}-M\right) \hat{\mathbf{n}} \times \mathbf{H}+\frac{i}{\omega \mu_{0}} P \hat{\mathbf{n}} \times \mathbf{E}=\mathbf{0} \\
& \left(\frac{1}{2}-M\right) \hat{\mathbf{n}} \times \mathbf{E}-\frac{i}{\omega \varepsilon_{0} \varepsilon_{r}} P \hat{\mathbf{n}} \times \mathbf{H}=\mathbf{0}
\end{aligned}
$$

$M_{0}$ and $P_{0}$ are the integral operators as defined by Martin and Ola [1993]. They write for a tangential density c

$$
\begin{gathered}
M_{0} \mathbf{c}(\mathbf{R})=\hat{\mathbf{n}} \times \mathbf{c u r l} \int_{\Sigma} G_{0} \mathbf{c}\left(\mathbf{R}^{\prime}\right) d S_{\mathbf{R}^{\prime}} \\
P_{0} \mathbf{c}(\mathbf{R})=\hat{\mathbf{n}} \times \text { curl curl } \int_{\Sigma} G_{0} \mathbf{c}\left(\mathbf{R}^{\prime}\right) d S_{\mathbf{R}^{\prime}}
\end{gathered}
$$

with $\mathbf{R}^{\prime}=\mathbf{r}^{\prime}+h\left(\mathbf{r}^{\prime}\right) \hat{\mathbf{z}}$ a point on the surface and expression

$$
G_{0}=-\frac{\exp \left(i K_{0}\left|\mathbf{R}-\mathbf{R}^{\prime}\right|\right)}{4 \pi\left|\mathbf{R}-\mathbf{R}^{\prime}\right|}
$$

for the free space Green's function. Operators $M$ and $P$ follow similar expressions, but with the wave number
$K=K_{0} \sqrt{\varepsilon_{r}}$ instead of $K_{0}$. The scattered field can be evaluated from $\hat{\mathbf{n}} \times \mathbf{E}$ and $\hat{\mathbf{n}} \times \mathbf{H}$ at any point or in far field.

[7] Due to the short coupling phenomenon for rough surface scattering [Maystre, 1983], the numerical support of the unknowns $\hat{\mathbf{n}} \times \mathbf{E}$ and $\hat{\mathbf{n}} \times \mathbf{H}$ is only some wavelengths larger than the support of the right hand sides $\hat{\mathbf{n}} \times \mathbf{E}^{i}$ and $\hat{\mathbf{n}} \times \mathbf{H}^{i}$. A tapered incident beam thus bounds the problem to a finite area of the interface. However, at LGI, the size of the beam's footprint on the surface increases as $\gamma_{0}^{-3}$ [Soriano et al., 2006] and the tapered beam turns useless.

[8] Therefore, we consider plane wave illumination in order to address LGI, and now following the GMoM, the surface $\Sigma$ is a local perturbation of the $(x O y)$ plane, with roughness over a given area. On Figure $1, L$ denotes the horizontal extension of the roughness. The idea is to find integral equations with right hand sides of numerical supports bounded to the perturbation. With an incident plane wave of wave vector $\mathbf{k}_{0}-q_{0} \hat{\mathbf{z}}$ and electric field

$$
\mathbf{E}^{i}=\mathbf{E}_{0} e^{i\left(\mathbf{k}_{0} \cdot r-q_{0} z\right)}
$$

of vector amplitude $\mathbf{E}_{0}$ and since the rough surface $\Sigma$ is a perturbation of the $(x O y)$ plane, it is interesting to define $\mathbf{E}^{r}$ and $\mathbf{E}^{t}$ the fields that would be reflected and transmitted by a plane interface $h(\mathbf{r})=0$. In this paper, reflected and transmitted fields will always refer to the solution of the scattering from the $(x O y)$ plane separating vacuum and scattering medium.

$$
\mathbf{E}^{r}=R \cdot \mathbf{E}_{0} e^{i\left(\mathbf{k}_{0} \cdot r+q_{0} z\right)} \quad \mathbf{E}^{t}=T \cdot \mathbf{E}_{0} e^{i\left(\mathbf{k}_{0} \cdot r-q_{0}^{\prime} z\right)}
$$

$R$ and $T$ are the reflection and transmission dyads built on the Fresnel coefficients. $-q_{0}^{\prime}$ the $z$ component of the transmitted wave vector $\mathbf{k}_{0}-q_{0}^{\prime} \hat{\mathbf{z}}$. It is defined by $q_{0}^{\prime}=\left(K_{0}^{2}\left(\varepsilon_{r}-1\right)+q_{0}^{2}\right)^{1 / 2}$ with $0 \leq \arg q_{0}^{\prime} \leq \pi / 2$.

[9] From now on, vector field $\mathbf{F}$ stands either for the electric field $\mathbf{E}$ or the magnetic field $\mathbf{H}$. The sole contribution from roughness to the total field in vacuum is $\mathbf{F}^{s}=\mathbf{F}-$ $\mathbf{F}^{i}-\mathbf{F}^{r}$. Hereafter $\mathbf{F}^{s}$ is called the scattered field, and its tangentials components $\mathbf{m}=\hat{\mathbf{n}} \times \mathbf{E}^{s}$ and $\mathbf{j}=\hat{\mathbf{n}} \times \mathbf{H}^{s}$ are solution of modified integral equations for the air

$$
\begin{aligned}
\left(\frac{1}{2}+M_{0}\right) \mathbf{j}-\frac{i}{\omega \mu_{0}} P_{0} \mathbf{m}=\mathbf{0} \\
\left(\frac{1}{2}+M_{0}\right) \mathbf{m}+\frac{i}{\omega \varepsilon_{0}} P_{0} \mathbf{j}=\mathbf{0}
\end{aligned}
$$

and for the lower medium.

$$
\begin{aligned}
\left(\frac{1}{2}-M\right) \mathbf{j}+\frac{i}{\omega \mu_{0}} P \mathbf{m}= & \left(\frac{1}{2}-M\right) \hat{\mathbf{n}} \times\left(\mathbf{H}^{i}+\mathbf{H}^{r}-\mathbf{H}^{t}\right) \\
& +\frac{i}{\omega \mu_{0}} P \hat{\mathbf{n}} \times\left(\mathbf{E}^{i}+\mathbf{E}^{r}-\mathbf{E}^{t}\right)
\end{aligned}
$$

$$
\begin{aligned}
\left(\frac{1}{2}-M\right) \mathbf{m}-\frac{i}{\omega \varepsilon_{0} \varepsilon_{r}} P \mathbf{j}= & \left(\frac{1}{2}-M\right) \hat{\mathbf{n}} \times\left(\mathbf{E}^{i}+\mathbf{E}^{r}-\mathbf{E}^{t}\right) \\
& -\frac{i}{\omega \varepsilon_{0} \varepsilon_{r}} P \hat{\mathbf{n}} \times\left(\mathbf{H}^{i}+\mathbf{H}^{r}-\mathbf{H}^{t}\right)
\end{aligned}
$$

This can be proven for example by considering the integral equations satisfied by the tangential components of $\mathbf{F}^{i}, \mathbf{F}^{r}$ 
and $\mathbf{F}^{t}$. By definition, $\hat{\mathbf{n}} \times\left(\mathbf{F}^{i}+\mathbf{F}^{r}-\mathbf{F}^{t}\right)=\mathbf{0}$ at any point of the $(x O y)$ plane, that is satisfying $z=0$ and $\hat{\mathbf{n}}=\hat{\mathbf{z}}$. As a consequence $\hat{\mathbf{n}} \times\left(\mathbf{E}^{i}+\mathbf{E}^{r}-\mathbf{E}^{t}\right)$ and $\hat{\mathbf{n}} \times\left(\mathbf{H}^{i}+\mathbf{H}^{r}-\mathbf{H}^{t}\right)$ both vanish outside the rough region, and even if $M$ and $P$ are integral operators, we can assume that the right hand sides of (12) and (13) do show bounded support. Finally, the four integral equations (10), (11), (12) and (13) can be combined in the system

$$
\begin{aligned}
& {\left[\begin{array}{cc}
1+M_{0-M} & -\frac{i}{\omega \mu_{0}}\left(P_{0}-P\right) \\
+\frac{i}{\omega \mu_{0}}\left(P_{0}-P\right) & \frac{1+\varepsilon_{r}}{2}+M_{0}-\varepsilon_{r} M
\end{array}\right]\left[\begin{array}{c}
\mathbf{j} \\
\mathbf{m}
\end{array}\right]} \\
& =\left[\begin{array}{cc}
\frac{1}{2}-M & +\frac{i}{\omega \mu_{0}} P \\
-\frac{i}{\omega \mu_{0}} P & \varepsilon_{r}\left(\frac{1}{2}-M\right)
\end{array}\right]\left[\begin{array}{c}
\hat{\mathbf{n}} \times\left(\mathbf{H}^{i}+\mathbf{H}^{r}-\mathbf{H}^{t}\right) \\
\hat{\mathbf{n}} \times\left(\mathbf{E}^{i}+\mathbf{E}^{r}-\mathbf{E}^{t}\right)
\end{array}\right]
\end{aligned}
$$

where the left-hand side matrix bears only weakly singular integral operators: $M, M_{0}$ and $P_{0}-P$ [Martin and Ola, 1993]. Operators $P_{0}$ and $P$ are hyper singular, since their kernels include the second derivative of the Green's function. They appear separately in the right-hand side of (14), but since they apply to fields with analytical expressions, one [Poggio and Miller, 1973] or two [Nédélec, 2001] derivatives can be transferred from the kernel to the fields. System (14) is designed for the numerical solution of the rough surface scattering problem at LGI, and can be discretized with low-order method of moment.

[10] Since $-q_{0}^{\prime}$ the vertical component of the transmitted wave vector has an imaginary part for lossy dielectrics and conductors, the transmitted field is exponentially decreasing for negative heights, but also exponentially increasing for positive heights. This leads to difficulties with the numerical solving of (14) in the case of very lossy dielectrics and rough surfaces with large height root mean squares. This integral system should be restricted to transparent or slightly lossy dielectrics. For numerous media [Senior and Volakis, 1995] the total electric and magnetic fields satisfy a boundary condition on the surface that is local, or can be assumed to be so. This impedance boundary condition is of general expression

$$
\hat{\mathbf{n}} \times \mathbf{E}=\hat{\mathbf{n}} \times Z_{h} \hat{\mathbf{n}} \times \mathbf{H}
$$

with $Z_{h}$ a local operator defined for the rough surface $z=h(\mathbf{r})$. It is assumed that $Z_{h}$ turns to a constant $Z_{0}$ on the $(x O y)$ plane, so that the reflected field is a plane wave for an incident plane wave and satisfy on the mean plane $(z=0 \Rightarrow \hat{\mathbf{n}}=\hat{\mathbf{z}})$ the relation

$$
\hat{\mathbf{z}} \times\left(\mathbf{E}^{\mathbf{i}}+\mathbf{E}^{\mathbf{r}}\right)=\hat{\mathbf{z}} \times Z_{0} \hat{\mathbf{z}} \times\left(\mathbf{H}^{\mathbf{i}}+\mathbf{H}^{\mathbf{r}}\right)
$$

With (15) and (16) included in (10) or (11), two single integral equations with unknown $\mathbf{j}$ are obtained. For example, (15) in (10) gives

$$
\begin{aligned}
& \left\{\left(\frac{1}{2}+M_{0}\right)-\frac{i}{\omega \mu_{0}} P_{0} \hat{\mathbf{n}} \times Z_{h}\right\} \mathbf{j} \\
& \quad=\frac{i P_{0} \hat{\mathbf{n}} \times}{\omega \mu_{0}}\left\{Z_{h} \hat{\mathbf{n}} \times\left(\mathbf{H}^{\mathbf{i}}+\mathbf{H}^{\mathbf{r}}\right)-\left(\mathbf{E}^{\mathbf{i}}+\mathbf{E}^{\mathbf{r}}\right)\right\}
\end{aligned}
$$

The relation (16) implies that the term $\hat{\mathbf{n}} \times\left\{Z_{h} \hat{\mathbf{n}} \times\left(\mathbf{H}^{\mathbf{i}}+\mathbf{H}^{\mathbf{r}}\right)-\right.$ $\left.\left(\mathbf{E}^{\mathbf{i}}+\mathbf{E}^{\mathbf{r}}\right)\right\}$ of the right hand side vanishes outside the rough region, thus auguring a bounded right-hand side for (17) or other integral equation in the impedance case. The perfectly conducting case corresponds to the trivial $Z_{h}=Z_{0}=0$, while the Leontovich boundary condition writes $Z_{h}=Z_{0}=\sqrt{\frac{\mu_{0}}{\varepsilon_{0} \varepsilon_{r}}}$. Surface impedance with curvature correction for the homogeneous complex dielectric lower medium is derived from Maxwell's equations in the work of Ong et al. [1994] and Marvin and Celli [1994], and is included in a rough surface scattering model for metals in Optics and the seawater at microwave frequency in the work of Soriano and Saillard [2001, 2003].

[11] To conclude this section, integral equations (14) and (17) allow the numerical computation of the tangential components of the electromagnetic field in a rough surface wave scattering problem with an incident plane wave and without edge effects.

\section{Scattered Field}

[12] Following the boundary integral formalism in homogeneous media, the electromagnetic field at any point is related to the tangential components of the field on the interface. With $\mathbf{F}^{s}=\mathbf{F}-\mathbf{F}^{i}-\mathbf{F}^{r}$ for $\mathbf{F}=\mathbf{E}, \mathbf{H}$ and $\mathbf{m}=\hat{\mathbf{n}} \times \mathbf{E}^{s}$, $\mathbf{j}=\hat{\mathbf{n}} \times \mathbf{H}^{s}$ and $G_{0}$ the free space Green's function for vacuum (7), it writes for the electric field

$$
\begin{aligned}
& -\frac{i}{\omega \varepsilon_{0}} \operatorname{curl} \operatorname{curl}\left(G_{0} * \mathbf{j} \delta_{\Sigma}\right) \\
& -\operatorname{curl}\left(G_{0} * \mathbf{m} \delta_{\Sigma}\right)=\left\{\begin{array}{r}
\mathbf{E}^{s}, z>h(\mathbf{r}) \\
\mathbf{0}, z<h(\mathbf{r})
\end{array}\right.
\end{aligned}
$$

with * the space convolution operation and $\delta_{\Sigma}$ de Dirac function associated to the surface. As soon as fictious currents $\mathbf{m}$ and $\mathbf{j}$ are of bounded numerical support, the scattered field writes in far-field condition as a spherical wave of vector amplitude $\mathbf{S}$. At point of the vacuum $\mathbf{R}_{0}=\mathbf{r}_{0}+z_{0} \hat{\mathbf{z}}$, $z_{0}>h\left(\mathbf{r}_{0}\right)$

$$
\mathbf{E}^{s}=\mathbf{E}-\mathbf{E}^{i}-\mathbf{E}^{r} \sim-i \frac{e^{i K_{0}\left|\mathbf{R}_{0}\right|}}{\left|\mathbf{R}_{0}\right|} \mathbf{S}^{+}(\mathbf{k})
$$

at the far-field limit for $\mathbf{K}\left|\mathbf{R}_{0}\right|=K_{0} \mathbf{R}_{0}$. One can define out of equation (18) the so-called vector scattering amplitude

$$
\begin{aligned}
\mathbf{S}^{ \pm}(\mathbf{k})= & \frac{1}{4 \pi} \int_{\Sigma}\left(-\mathbf{K} \times \mathbf{m}(\mathbf{R})+\frac{1}{\omega \varepsilon_{0}} \mathbf{K} \times(\mathbf{K} \times \mathbf{j}(\mathbf{R}))\right) \\
& \cdot \exp (-i \mathbf{K} \cdot \mathbf{R}) d S_{\mathbf{R}}
\end{aligned}
$$

associated to the wave vector $\mathbf{K}=\mathbf{k} \pm q \hat{\mathbf{z}}$ with $\mathbf{K}^{2}=\mathbf{k}^{2}+$ $q^{2}=K_{0}^{2}$. The Normalized Radar Cross Section (NRCS) is proportional to the square modulus of that scattering amplitude. Since formula (18) is zero under the surface, $\mathbf{S}^{-}$vanishes for all $\mathbf{k}$.

$$
\mathbf{S}^{-}(\mathbf{k})=0
$$


This property is generally referred to as the extinction theorem: $\mathbf{m}$ and $\mathbf{j}$ are fictitious surface currents in air that do not radiate under the surface. As a consequence, the scattering amplitude in the upper half-space $\mathbf{S}^{+}(\mathbf{k})$ can also be written as the difference

$$
\mathbf{S}^{d}(\mathbf{k})=\mathbf{S}^{+}(\mathbf{k})-\mathbf{S}^{-}(\mathbf{k})
$$

Expressions $\mathbf{S}^{+}(\mathbf{k})$ and $\mathbf{S}^{d}(\mathbf{k})$ are theoretically equivalent, but reveal their difference when numerically evaluated at LGS. This can be understood by comparing the term from (20) $\exp (-i q h(\mathbf{r})) \sim 1$ at grazing $q \rightarrow 0$, which turns in (22) to

$$
\exp (-i q h(\mathbf{r}))-\exp (-i q h(\mathbf{r}))=-2 i \sin (q h(\mathbf{r}))=O(q)
$$

using Landau notation. Hence, in a numerical computation, expression $\mathbf{S}^{+}(\mathbf{k})$ would generally lead to a nonzero value at $q=0$, while expression $\mathbf{S}^{d}(\mathbf{k})$ would meet a $O(q)$ behavior at LGS. The choice between $\mathbf{S}^{+}(\mathbf{k})$ and $\mathbf{S}^{d}(\mathbf{k})$ should thus be funded on theoretical arguments.

[13] Since the work by Tatarskii and Charnotskii [1998a, 1998b] on scalar waves, we know that the asymptotic behavior of the scattered far-field at LGI and/or LGS can be theoretically predicted. Moreover, LGI and LGS behaviors are simply connected through the Lorenz reciprocity theorem. At LGI, the limit is indicated by the right-hand side of the relevant integral equations (14) or (17). The reasoning is that integral operators and scattering formula don't depend on the incident field and $q_{0}$. Consequently, the behavior of the right hand side is transferred to the unknowns $\mathbf{m}$ and $\mathbf{j}$ through the integral equation and then to the scattering amplitude. From the Fresnel's coefficients for a finite permittivity or conductivity, it can be easily shown that the reflection and transmission dyads $R=-I+O\left(q_{0}\right)$ and $T=O\left(q_{0}\right)$, with Landau notation, and $I$ the dyadic identity. Then $\mathbf{E}^{i}+\mathbf{E}^{r}-\mathbf{E}^{t}=O\left(q_{0}\right)$ and $\mathbf{H}^{i}+\mathbf{H}^{r}-\mathbf{H}^{t}=O\left(q_{0}\right)$, with final general result that $\mathbf{S}^{+}(\mathbf{k})=O\left(q_{0} q\right)$, with the LGS behavior obtained by reciprocity. This holds in the impedance case, as long as $R=-I+O\left(q_{0}\right)$ is met. A notable exception is the perfectly conducting surface: when the permittivity is first made infinite, or equivalently $Z_{h}=Z_{0}=0$, the reflection coefficient for the $\mathrm{V}$ incident polarization is +1 for any angle. The limit $\mathbf{S}^{+}(\mathbf{k})=O\left(q_{0}\right)$ at LGI is thus lost, and as explained by Spiga et al. [2008], the perfectly conducting model must be discarded in $\mathrm{V}$ polarization for incidence angles around or larger than the Brewster angle, with reciprocal situation for the $\mathrm{V}$ component of the scattering amplitude at LGS.

[14] Therefore, it appears that the boundary integral formalism (14) and (17) is also a theoretical tool to predict the behavior of the scattering amplitude for rough surface scattering problem at low-grazing angles. With this knowledge, one can numerically evaluate the scattering amplitude from the surface unknowns $\mathbf{m}$ and $\mathbf{j}$ through a formula that enforce its theoretical behavior at LGS. When the rough interface separates vacuum and an homogeneous medium with finite permittivity, the scattering amplitude show a $\mathbf{S}^{+}(\mathbf{k})=O\left(q_{0}\right)$ theoretical limit at LGI. Numerically fitting this behavior with formula (20) requires accurate cancelation of contributions from all over the rough area. It will be proved in section 5 that the scattering amplitude predicted by formula (22) coincides with the more classical
(20) at non-grazing scattering angle and ensures the correct asymptotic behavior at LGS.

\section{Extrapolation}

[15] Solving such scattering problems, even with fast numerical solvers, remains time consuming. In addition, the iterative solvers do not take benefit from the solution at a given incidence angle to speed up the computation for another incidence angle.

[16] With the aim of saving computation time, we show how a single computation of the backscattered field for a given grazing incidence can be extrapolated from the knowledge of the theoretical asymptotic behavior of the scattering amplitude. (17) and (14) are linear integral equation or system that we symbolically denote

$$
\mathbf{A X}=\mathbf{B}
$$

The integral operators, $M_{0}, P_{0}$ and so on, depend on the roughness of the surface and boundary conditions, but not on the incident field. As such, $\mathbf{A}$ is not a function of $\gamma_{0}$ or $q_{0}$. Equation (23) can be derived with respect to $q_{0}$, and at the LGI limit

$$
\mathbf{A}\left(\frac{\partial \mathbf{X}}{\partial q_{0}}\right)_{q_{0}=0}=\left(\frac{\partial \mathbf{B}}{\partial q_{0}}\right)_{q_{0}=0}
$$

This linear equation can be solved to compute $\left(\frac{\partial \mathbf{m}}{\partial q_{0}}\right)_{q 0=0}$ and $\left(\frac{\partial \mathbf{j}}{\partial q_{0}}\right) q 0=0$.

[17] Since the scattering amplitude depends on $q_{0}$ only through $\mathbf{m}$ and $\mathbf{j}$, the derivative $\left(\frac{\partial \mathbf{S}^{ \pm}}{\partial q_{0}}\right) q 0=0$ follows the same expression (20) as $\mathbf{S}^{ \pm}$, with only $\mathbf{m}$ and $\mathbf{j}$ replaced by their LGI derivatives $\left(\frac{\partial \mathbf{m}}{\partial q_{0}}\right)_{q 0}=0$ and $\left(\frac{\partial \mathbf{j}}{\partial q_{0}}\right)_{q 0=0}$, respectively.

[18] Denoting by $\sigma_{h}$ the RMS height of the rough surface, a first-order expansion

$$
\mathbf{S} \simeq q_{0}\left(\frac{\partial \mathbf{S}}{\partial q_{0}}\right)_{q_{0}=0}
$$

is expected to be accurate as far the product $q_{0} \sigma_{h}$ remains small.

\section{Numerical Results}

[19] Here, the integral equation or system derived in section 2 is solved with the help of the method of moments. Basis and testing functions are pulse (piecewise constant) and Dirac functions, respectively. The linear system stemming from discretization, with its matrix full, is far too large for direct algorithms. Its solution is based on a fast iterative scheme. With the multilevel canonical grid method [Li et al., 2001; Soriano and Saillard, 2003], the matrix-vector product is performed at floating point operation (CPU) and central memory (RAM) costs that behave as $N \log _{2} N$ and $N$, respectively, where $N$ is the number of unknowns. The system is solved with a preconditioned GMRES.

[20] The first example aims at pointing out the advantage of the approach used here. For this purpose, we consider the sea surface described by a fully developed spectrum [Elfouhaily et al., 1997], with wind speed $3 \mathrm{~m} / \mathrm{s}$. To speed up the computation, it is assumed to be perfectly conducting. 


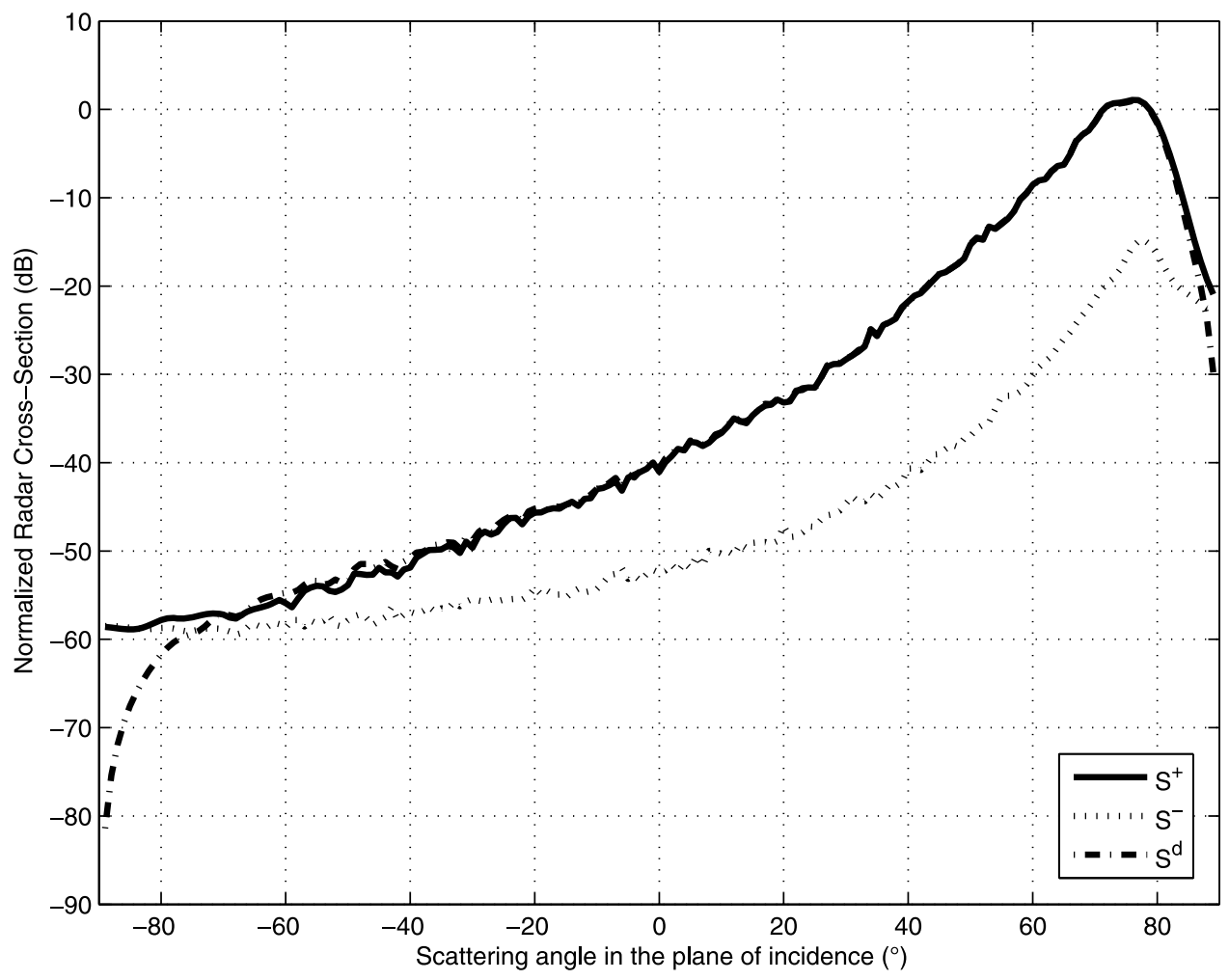

Figure 2. $\mathrm{HH}$ component of the bistatic normalized scattering cross section at $\mathrm{L}$ band $(1.2 \mathrm{GHz})$ and $89^{\circ}$ incidence versus scattering angle in the plane of incidence for sea surface with wind speed $3 \mathrm{~m} / \mathrm{s}$. The surface is assumed to be perfectly conducting. The NRCS is derived from three different scattering amplitude expressions $\mathbf{S}^{+}$(solid line), $\mathbf{S}^{-}$(dotted line) and the difference $\mathbf{S}^{d}=\mathbf{S}^{+}-\mathbf{S}^{-}$(dash-dotted line).

The HH bistatic NRCS at L band (1.2 GHz, wavelength $\lambda=25 \mathrm{~cm})$ in the plane of incidence at $89^{\circ}$ incidence, has been computed through averaging over 100 samples, each of them being $12 \mathrm{~m}=48 \lambda$ square. The surface is sampled with a one-eighth wavelength step; an associated $4 K_{0}$ high frequency cut-off is applied to the sea spectrum. The roughness is limited to the central part of the samples, with $2 \lambda$-long plateaus on the edges: this means with notations of Figure 1 that $L_{g}=L_{d}=2 \lambda$ and $L=44 \lambda$. Note also that a smooth transition is applied between the roughness and the plateaus, over $4 \lambda$. As a consequence, the area $A$ for the normalization of the RCS is difficult to define for the GMoM. Here, it is set to $A=40 \lambda \times 40 \lambda$. This may have a little impact on comparisons to other approaches or experimental data. All those dimensions are chosen with no reference to the incidence angle. Still, for the Monte Carlo average to be statistically significant, the length of the rough part of the samples has to be much larger than the characteristic horizontal scales of the roughness, that is the wavelength of the peak wave in the ocean surface case. Three curves are plotted in Figure 2: one represents the bistatic NRCS as derived from Stratton-Chu formula $\left(\mathbf{S}^{+}\right)$, one is the NRCS in the lower medium, which, according to the extinction theorem, should vanish $\left(\mathbf{S}^{-}\right)$and the third one is the NRCS derived from the difference $\mathbf{S}^{d}=\mathbf{S}^{+}-\mathbf{S}^{-}$. As usual, the angular resolution of those diagrams is inversely proportional to the samples dimensions. In order for rapid angular variations in radar cross sections to be studied, one has to correctly set the surface size, and to test convergence on this parameter.

[21] Clearly, the use of Stratton-Chu formula $\mathbf{S}^{+}$does not provide an accurate estimation of the bistatic cross section beyond $-75^{\circ}$. The ratio between the largest and the smallest value of the NRCS, which can be seen as the numerical dynamic range, does not exceed $10^{6}$. It is linked to the accuracy with which the extinction theorem $\mathbf{S}^{-}$is satisfied. Performing the difference (22) permits to significantly improve the dynamic range and to compute the NRCS at grazing scattering angles.

[22] We now test the performances of first orders approximate surface scattering methods at low-grazing angles. Since the Kirchhoff approximation of Beckmann and Spizzichino [1963] fails to show the correct $q q_{0}$ behavior at grazing, it is discarded and we focus on the small perturbation method (SPM1) of Rice [1951] and the small slope approximation (SSA1) of Voronovich [1994]. There exist for such theories statistical formulations associated to a plane wave illuminating an infinite surface with stationary roughness. In order to avoid Monte Carlo and normalization errors, and to focus on the impact of physical assumptions on the scattering at grazing, approximate models NRCS are computed through Monte Carlo average, with the same samples and configuration as for the GMoM. Still considering the ocean surface at $\mathrm{L}$ band and $89^{\circ}$ incidence, we compare on Figure 3 the bistatic diagrams of the GMoM and SSA1. The standard validity criterions are $\sigma_{h}<\lambda / 20$ for SPM1 and $K_{0} \sigma_{h} \sigma_{s}<1$ for SSA1, with $\sigma_{h}$ and $\sigma_{s}$ the surface 


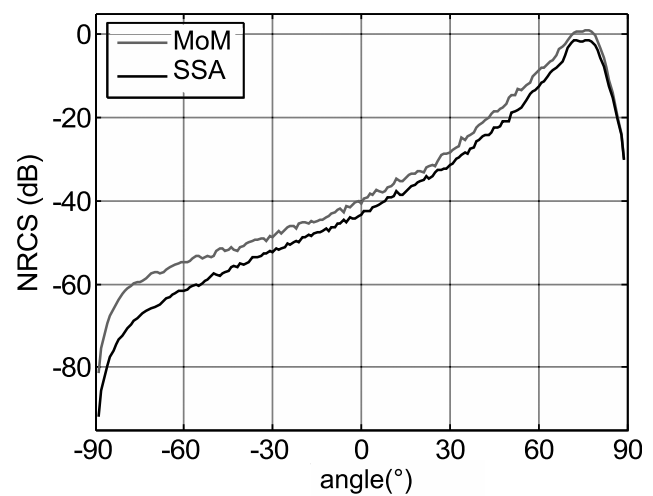

Figure 3. HH component of the bistatic normalized scattering cross section predicted by the GMoM (dashed line) and SPM1 or SSA1 (solid line) at L band $(1.2 \mathrm{GHz})$ and $89^{\circ}$ incidence versus scattering angle in the plane of incidence for sea surface with wind speed $3 \mathrm{~m} / \mathrm{s}$. The surface is assumed to be perfectly conducting.

height and slope root mean squares. Our surface is outside SPM1's validity domain, but inside SSA1's, with $K_{0} \sigma_{h} \sigma_{s}=$ 0.16 . Nevertheless, on this example, the SPM1 diagram do coincide perfectly with SSA1 on the whole scattering angles range, and thus is not plotted. It can be seen that the approximate models underestimate the NRCS by several $\mathrm{dBs}$, with the error increasing from $2 \mathrm{~dB}$ at $+80^{\circ}$ to $8 \mathrm{~dB}$ in low-grazing backward angles and in the backscattering direction.

[23] To extend this study, we now consider rough surfaces with a Gaussian spectrum of correlation radius $\lambda / 2$ and a height root mean square varying from $0.08 \lambda$ to $0.83 \lambda$, in the perfectly conducting case. With such a short correlation radius, the sample side can be shortened to $16 \lambda$, with $2 \lambda$ plateaus, still. The number of samples for the Monte Carlo average is now 200. In Figure 4, the HH monostatic NRCS difference between SSA1 and the GMoM is plotted against the surfaces height root mean square for angles $20^{\circ}, 60^{\circ}, 80^{\circ}$ and $89^{\circ}$. If the SPM1 enjoys a statistical expression for its NRCS, the GMoM mean NRCS is obtained through Monte Carlo average on a finite number of samples. Corresponding error bars appear on plots of Figure 4. At the small height limit, the curves for all angles tend toward zero, thus underlining the accuracy of the GMoM. Then, the SSA1 error increases more or less linearly with the height root mean square, but with slopes that strongly depend on the angle. For a height of $\lambda / 20$, its error is smaller that $1 \mathrm{~dB} 20^{\circ}$ angle, around $3 \mathrm{~dB}$ at $60^{\circ}$, but reaches $8 \mathrm{~dB}$ at $80^{\circ}$ and even $10 \mathrm{~dB}$ at $89^{\circ}$. For that Gaussian surfaces, SPM1 and SSA1 do not coincide exactly, still the results and conclusions are similar. The GMoM clearly states that the domain of validity of approximate methods varies with the angle and narrows at grazing. Beyond $80^{\circ}$, the $3 \mathrm{~dB}$ error line is crossed as soon as $\lambda / 60$ height root mean square for both SPM1 and SSA1.

[24] Up to now, bistatic diagrams have been preferently presented, since with the Method of Moment, for a given incidence angle, it is easy and fast to compute through formula (20) the scattered field for any (reasonable) number of scattering angles, but when the incidence is varied, the integral equation (17) or system (14) is to be solved for a new right-hand side. When direct methods such as LU decomposition are used to solve the linear system that comes from the discretization of integral equations, multiple right-hand sides are not a problem. However, the number of unknowns $N$ is too large for today's computers, except for one-dimensional (1D) surfaces, i.e. with an invariance along one horizontal direction. Beyond this assumption, the system is solved iteratively, and the computing time is

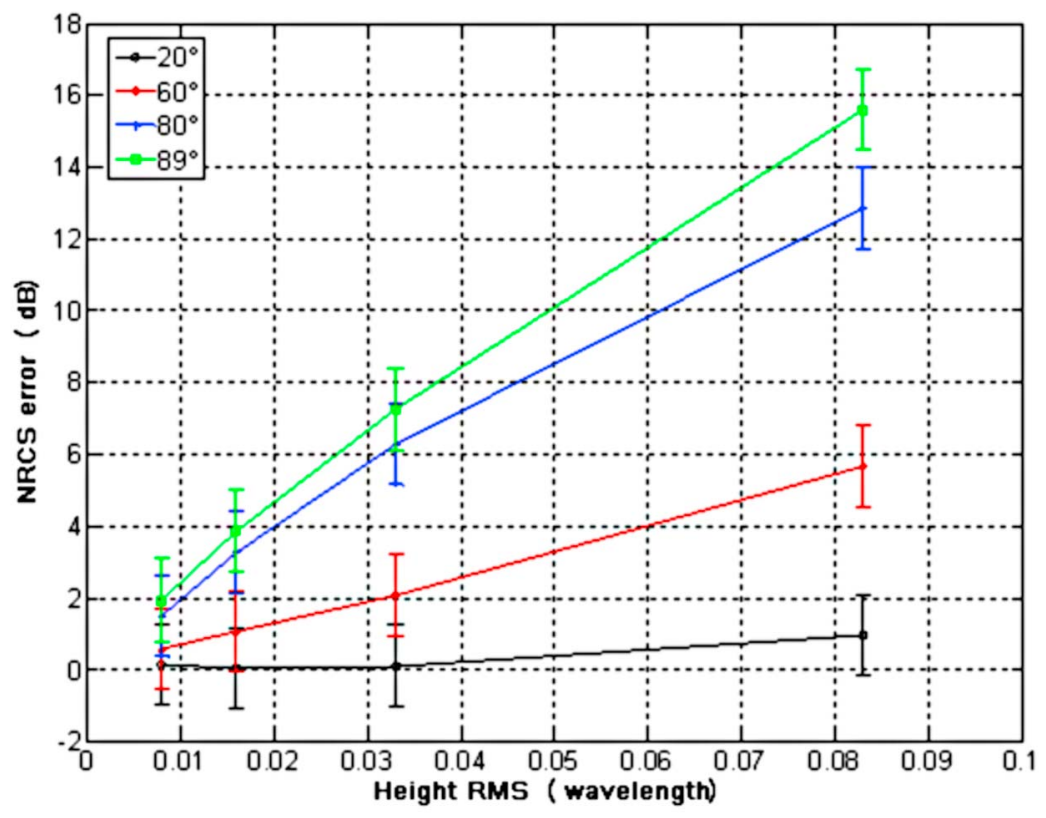

Figure 4. HH monostatic NRCS error in the plane of incidence between SPM1 and the GMoM versus the surfaces height root mean square for perfectly conducting surfaces with Gaussian spectrum of correlation radius $\lambda / 2$ and angles $20^{\circ}, 60^{\circ}, 80^{\circ}$ and $89^{\circ}$, with error bars for the GMoM Monte Carlo average. 


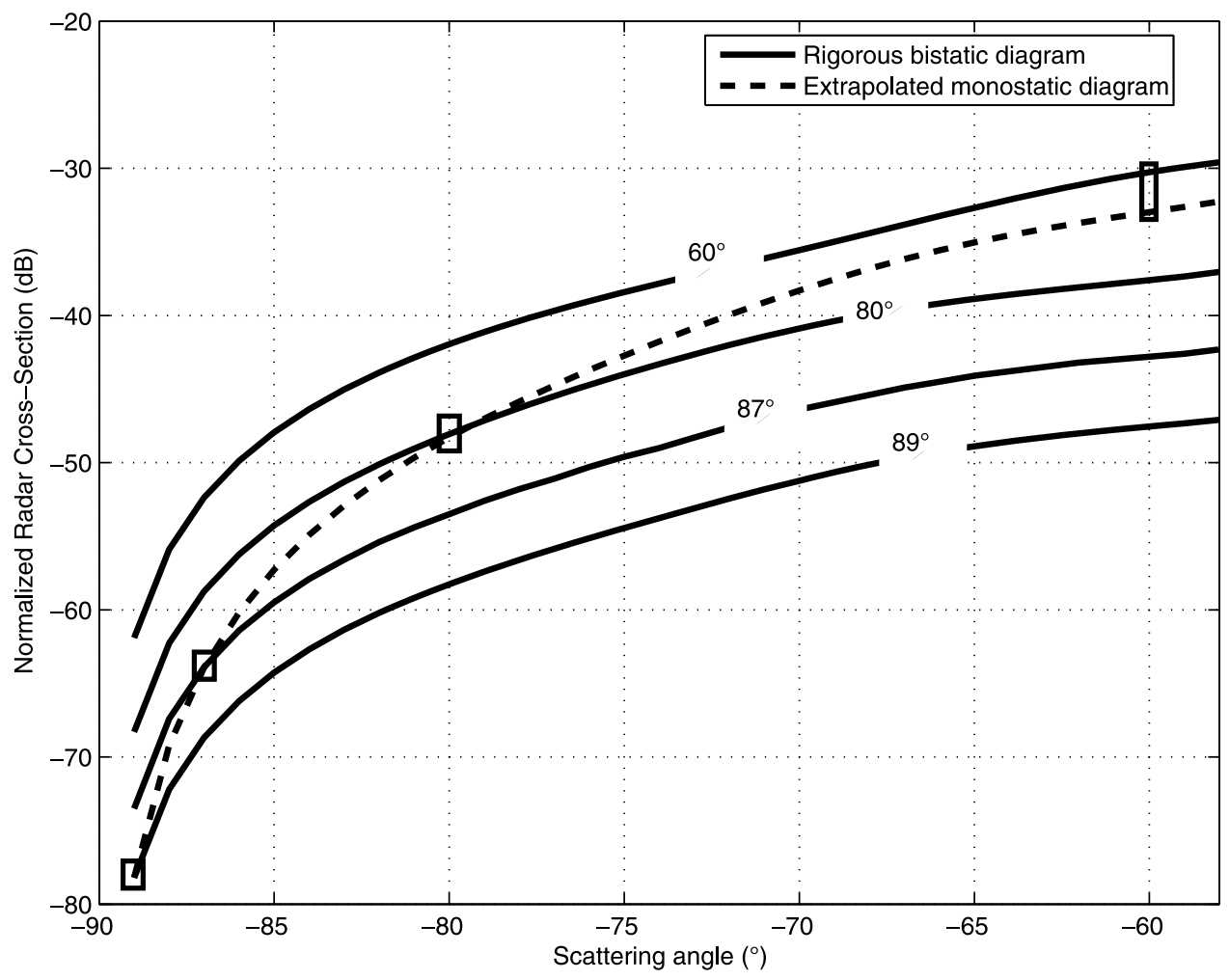

Figure 5. HH bistatic NRCS for incidence angles $60^{\circ}, 80^{\circ}, 87^{\circ}$ and $89^{\circ}$ (solid lines) and extrapolated $\mathrm{HH}$ monostatic NRCS (dashed line) for perfectly conducting surfaces with Gaussian spectrum of height root mean square $0.83 \lambda$ and correlation radius $\lambda / 2$ versus scattering angle in the plane of incidence.

proportional to the number of incidence angles. For the monostatic diagram in the low-grazing angles region, one can try extrapolation (25). On Figure 5 is plotted for the rougher Gaussian surface the bistatic NRCS at backward scattering angles for incidence angles $60^{\circ}, 80^{\circ}, 87^{\circ}$ and $89^{\circ}$, and the NRCS obtained through (25). It appears that extrapolation matches the rigorous computation at angles $89^{\circ}$ and $87^{\circ}$. At $80^{\circ}$, a discrepancy starts being visible, but the error is smaller than $1 \mathrm{~dB}$, and obviously the extrapolation looses accuracy at larger grazing angles, with a 3.5 $\mathrm{dB}$ error at $60^{\circ}$.

\section{Conclusion}

[25] The incidence angle is no longer a limit to the numerical solution of the rough surface electromagnetic wave scattering problem. We have presented a grazing surface integral formalism with a numerical cost made independent of the incidence. The cases of a rough interface between two homogeneous media as well as the impedance boundary condition are detailed in full vector theory. In this paper, the model has been implemented following the Method of Moments with pulse basis functions and Dirac testing functions, a classical scheme. As a matter of fact, the integral equations are modified, but the integral operators being left untouched, the countless associated numerical techniques published in the literature for discretization, such as the Boundary Element Method [Rao et al., 1982; Chew et al., 2008], or acceleration techniques such as the Forward
Backward Spectral Acceleration of Torrungrueng and Johnson [2001] or the Fast Multipole Method of Jandhyala et al. [1998], might be used as is.

[26] Also, the scattering amplitude formula shows an alternative equivalent expression that reveals best fitted to low-grazing scattering angles. The presented surface integral formalism combined with the equivalent scattering formula constitute a dedicated model for the wave scattering from rough surfaces at low-grazing incidence and scattering angles.

[27] Tested first-order approximate methods, namely the small perturbation method and the small slope approximation perform very poorly at low-grazing angles, with their validity domain drastically narrowed. At grazing, multiple scattering is often modelized by a shadowing function [Bourlier et al., 2002]. However, it appears that first-order approximations systematically underestimate the Radar Cross Section at grazing angles, and thus applying a shadowing function would only increase the gap between rigorous and approximate computations.

[28] Nevertheless, the grazing angles regime also have positive characteristics. It has been shown how the monostatic diagram at large incidence angles can be extrapolated from a single (statistical) calculus at grazing incidence. This is a lead for the development of approximate scattering models devoted to the low-grazing angles.

[29] Acknowledgments. This work was partially supported by 


\section{References}

Beckmann, P., and A. Spizzichino (1963), The Scattering of Electromagnetic Waves From Rough Surfaces, Int. Ser. Monogr. Electromagn. Waves, vol. 4, Pergamon, New York.

Bourlier, C., G. Berginc, and J. Saillard (2002), One-and twodimensional shadowing functions for any height and slope stationary uncorrelated surface in the monostatic and bistatic configurations, IEEE Trans. Antennas Propag., 50, 312-324.

Braunisch, H., Y. Zhang, C. O. Ao, S. E. Shih, Y. E. Yang, K. H. Ding, and J. A. Kong (2000), Tapered wave with dominant polarization stat for all angles of incidence, IEEE Trans. Antennas Propag., 48, 1086-1096.

Chew, W., M. Tong, and B. Hu (2008), Integral Equation Methods for Electromagnetic and Elastic Waves, Morgan and Claypool, San Rafael, Calif.

Colton, D., and R. Kress (1983), Integral Equations in Scattering Theory, Wiley-Intersci., New York.

Elfouhaily, T., B. Chapron, K. Katsaros, and D. Vandemark (1997), A unified directional spectrum for long and short wind-driven waves, J. Geophys. Res., 102(C7), 15,781-15,796.

Jandhyala, V., B. Shanker, E. Michielssen, and W. C. Chew (1998), Fas algorithm for the analysis of scattering by dielectric rough surfaces, J. Opt. Soc. Am. A, 15, 1877-1885.

Li, S. Q., C. H. Chan, M. Y. Xia, B. Zhang, and L. Tsang (2001), Multilevel expansion of the sparse-matrix canonical grid method for twodimensional random rough surfaces, IEEE Trans. Antennas Propag. 47, 752-763.

Martin, P. A., and P. Ola (1993), Boundary integral equations for the scattering of electromagnetic waves by a homogeneous dielectric obstacle Proc. R. Soc. Edinburgh, 123A, 185-208.

Marvin, A. M., and V. Celli (1994), Relation between the surface impedance and the extinction theorem on a rough surface, Phys. Rev. B, $50,14,546-14,553$.

Maystre, D. (1983), Electromagnetic scattering from perfectly conducting rough surfaces in the resonance region, IEEE Trans. Antennas Propag., $31,885-895$.

Nédélec, J. (2001), Acoustic and Electromagnetic Equations: Integral Representations for Harmonic Problems, Springer, New York.

Ong, T., V. Celli, and A. Marvin (1994), General relation between surface impedance and surface curvature, J. Opt. Soc. Am. A, 11, 759-765.

Poggio, A., and E. Miller (1973), Integral equation solutions of threedimensional scattering problems, in Computer Techniques for Electromagnetics, edited by R. Mittra, Permagon, Elmsford, N. Y.

Rao, S., D. Wilton, and A. Glisson (1982), Electromagnetic scattering by surfaces of arbitrary shape, IEEE Trans. Antennas Propag., 30, 409-418.

Rice, S. (1951), Reflection of electromagnetic waves from slightly rough surfaces, Commun. Pure Appl. Math., 4(2-3), 351-378.

Senior, T., and J. Volakis (1995), Approximate Boundary Conditions in Electromagnetics, Inst. of Eng. and Technol., Stevenage, U. K.
Soriano, G., and M. Saillard (2001), Scattering of electromagnetic waves from two-dimensional rough surfaces with impedance approximation, J. Opt. Soc. Am. A, 18, 124-133.

Soriano, G., and M. Saillard (2003), Modelization of the scattering of electromagnetic waves from the ocean surface, Prog. Electromagn. Res., 10, $101-128$.

Soriano, G., M. Joelson, and M. Saillard (2006), Doppler spectra from a two-dimensional ocean surface at L-band, IEEE Trans. Geosci. Remote Sens., 44(9), 2430-2437.

Soriano, G., P. Spiga, and M. Saillard (2010), Low-grazing angles scattering of electromagnetic waves from one-dimensional natural surfaces: Rigorous and approximate theories, C. R. Phys., 11(1), 77-86.

Spiga, P., G. Soriano, and M. Saillard (2008), Scattering of electromagnetic waves from rough surfaces: A boundary integral method for low-grazing angles, IEEE Trans. Antennas Propag., 56, 2043-2050.

Tatarskii, V., and M. Charnotskii (1998a), Universal behaviour of scattering amplitudes for scattering from a plane in an average rough surface for small grazing angles, Waves Random Complex Media, 8(1), 29-40.

Tatarskii, V., and M. Charnotskii (1998b), On the universal behavior of scattering from a rough surface for small grazing angles, IEEE Trans. Antennas Propag., 46, 67-72.

Toporkov, J. V., R. S. Awadallah, and G. S. Brown (1999), Issues related to the uses of a Gaussian-like incident field for low-grazing-angle scattering, J. Opt. Soc. Am. A, 16, 176-186.

Torrungrueng, D., and J. T. Johnson (2001), The forward-backward method with a novel spectral acceleration algorithm (FB/NSA) for the computation of scattering from two-dimensional large-scale impedance random rough surface, Microwave Opt. Tech. Lett., 29, 232-236.

Tsang, L., J. A. Kong, K. H. Ding, and C. O. Ao (2001), Scattering of Electromagnetic Waves: Numerical Simulations, Remote Sens. Ser. Wiley-Intersci., New York

Voronovich, A. G. (1994), Wave Scattering From Rough Surfaces, Springer, Berlin.

Wagner, R. L., J. Song, and W. C. Chew (1997), Monte-Carlo simulation of electromagnetic scattering from two-dimensional random rough surfaces, IEEE Trans. Antennas Propag., 45, 235-245.

West, J. (2003), On the control of edge diffraction in numerical rough surface scattering using resistive tapering, IEEE Trans. Antennas Propag., $51,3180-3183$.

Zhao, Z., L. Li, J. Smith, and L. Carin (2005), Analysis of scattering from very large three-dimensional rough surfaces using MLFMM and raybased analyses, IEEE Trans. Antennas Propag., 47, 20-30.

M. Saillard, Laboratoire de Sondage Electromagnétique de l'Environnement Terrestre, Université du Sud Toulon-Var, F-83957 La Garde, CEDEX, France. (saillard@univ-tln.fr)

G. Soriano, Sondage ElectroMagnétique et Optique, Institut Fresnel, Aix-Marseille Université, Campus de St Jérôme, F-13397 Marseille, CEDEX, France. (gabriel.soriano@fresnel.fr) 